\title{
Gross Hematuria: Assessment and Management at the End of Life
}

\author{
Hunter Groninger, MD ${ }^{1}$ and Jayne M. Phillips, MS, CRNP, OCN \\ ${ }^{1}$ Pain and Palliative Care Service, National Institutes of Health Clinical Center, Bethesda, MD
}

\begin{abstract}
A distressing complication for patients and families, gross hematuria at the end of life challenges hospice and palliative care clinicians to utilize skills in medical and nursing management, communication and clarification of patient goals, and relief of symptom burden. Massive hemorrhage in the genitourinary tract can radically alter the terminal trajectory for patients and necessitate intensive interventions aimed at promoting comfort. Here, a case of gross hematuria in an adult hospice patient serves to broaching decision-making challenges and management strategies.
\end{abstract}

\section{Keywords}

gross hematuria; hospice; palliative; pain management; urology; goals of care

Gross hematuria at the end of life constitutes a highly distressing complication for patients and families. To provide best supportive care in these cases, clinicians must combine skills in medical and nursing management, communication and clarification of patient goals, and relief of symptom burden. When hematuria progresses to massive hemorrhage in the genitourinary tract, the consequences can be catastrophic, often radically altering the terminal trajectory for patients and requiring intensive interventions aimed at promoting comfort. Here, we present a case of gross hematuria in an adult patient as a springboard to broaching decision-making challenges and management strategies relevant to the hospice and palliative medicine (HPM) provider. In this article, microscopic hematuria, pediatricspecific issues, and urologic disease-modifying care will not be addressed.

CASE (Part I): Mr. J.R. was a 72 year old with metastatic prostate cancer admitted from home to a freestanding inpatient hospice unit to manage cancer pain and hyperactive delirium. After 2 days, his pain appeared controlled with subcutaneous opiates and corticosteroids. A simple workup of his delirium (including medication review and urinalysis but no imaging) did not yield an etiology, but his agitation improved significantly with good pain control. Plans were made to discharge the patient home again with hospice support. On the night before discharge, he experienced red-tinged urine output. The hospice physician on-call asked that an indwelling Foley catheter be placed; the hospice nurse was able to place a coudé

Corresponding Author: Hunter Groninger, MD, Staff Clinician, Pain and Palliative Care, Clinical Center, National Institutes of Health, Building 10, Room 2-1733 MSC 1517, Bethesda, MD 20892, Tel: 301/594-9768, hunter.groninger@ nih.gov. 
catheter which the nurse chose to use in light of possible urinary obstruction in an elderly metastatic prostate patient.

Gross hematuria is first suspected with the presence of red or brown-tinged urine. As little as $1 \mathrm{~mL}$ of blood can produce a visible color change in $1 \mathrm{~L}$ of urine ${ }^{1}$; the deepness of color does not correlate with the amount of blood loss, although color can give helpful hints about briskness of bleeding and whether the clot is old or new. A few processes, such as medications, certain ingested foods and some benign medical conditions, promote a reddish urine color without the actual presence of red blood cells. Urinalysis dipstick and microscopy of a specimen can help diagnose gross hematuria and begin to distinguish possible etiologies [Figure 1]; this inexpensive test, readily available in hospital and clinic settings, can also be ordered for patients in home and inpatient hospice settings. In most cases, however, the presence of frank blood or clots in urine obviates the need for such testing. When new hematuria presents with fever, delirium, or new pain (symptoms already common in patients with advanced illnesses), a urine culture can help rule out an infectious etiology. Anticoagulated patients still require an appropriate workup since the hematuria will uncover an identifiable lesion in up to $25 \%$ of patients ${ }^{2}$.

Once the diagnosis of gross hematuria has been established, initial evaluation is aimed at delineating the anatomic origin of the hematuria along the genitourinary (GU) tract. [Table 1] Although the differential diagnosis may be lengthy, in the setting of advanced illness, often a careful history and focused physical examination can uncover probable sources.

[Table 2] Transient hematuria may more likely be from infection, nephrolithiasis, or trauma; persistent hematuria suggests glomerular disease and, especially in older individuals, malignancy or benign prostatic hypertrophy.

In most clinical circumstances, progressive or recurrent gross hematuria necessitates prompt urologic consultation and subsequent evaluation with imaging studies - options include cystoscopy, intravenous or retrograde pyelography, ultrasonography, magnetic resonance imaging and/or urography, and conventional or multidetector computed tomography (CT) imaging. Currently, there are no evidence-based guidelines as to optimal imaging modalities. However, for a complete evaluation of the entire GU tract, many experts recommend a combination of cystoscopy (to best view urethra, prostate, bladder and ureteral orifices) and CT urography (for ureters and kidneys) ${ }^{3,4}$. Urological and HPM clinicians alike should note that intraurethral instillation of lidocaine gel appears to improve moderate to severe pain control in patients undergoing flexible cystoscopy, but delay before scope insertion does not improve comfort ${ }^{5,6}$. For the patient with advanced illness, consideration of more extensive imaging or invasive workup must be balanced closely with the patient's goals of care, symptom burden, and overall prognosis.

Concomitant with the initial workup, clinicians must immediately assess severity of bleeding and facilitate patency of urinary outflow; urethral obstruction remains the major potential emergency in gross hematuria. ${ }^{4}$ In most cases, aspirin, nonsteroidal antiinflammatory drugs, anticoagulation and antiplatelet therapies should be held, at least temporarily while the patient is stabilized. Monitoring for hemodynamic instability with intravenous fluid infusion or red blood cell transfusions as indicated may be necessary; 
however, for many patients with advanced disease, such interventions may be inappropriate given clarified goals of care, or simply cannot be administered in a given care setting (e.g. home, long term care, inpatient hospice).

Case (Part II): At the inpatient hospice unit, small clots appeared in the urine, but otherwise, a physical examination was unremarkable. After several hours, repeat intermittent catheter irrigation yielded gross blood clots obstructing urine flow. Gradually, the patient became more agitated and required additional doses of pain medication which partly relieved his distress. The hospice nurse continued manual irrigation through the Foley catheter for the next hour.

For patients with patent urethras, Foley catheterization may be avoided and urine outflow can be monitored. ${ }^{4}$ When gross hematuria leads to clot formation and retention, control of urinary outflow may become challenging at best. Clinicians should initiate manual irrigation with the largest Foley catheter tolerable to the patient (usually 22-24 French). Currently, there are no evidence-based guidelines as to bladder irrigation strategies and nurses will need to consult with their individual agency or institution for standard operating procedures on CBI. Some experts advise a continuous bladder normal saline irrigation by gravity alone to achieve clear or pink urine ${ }^{7}$. Due to inter-individual variability in bladder size and anatomy, intra-abdominal pressure, and position, CBI can be most safely and optimally administered at a rate that establishes clear to light pink urine rather than at a set hourly rate, The nurse should monitor urine color, presence of clots, patency, absence of leaking around catheter, and bowel function (as constipation can worsen pain) to assess irrigation effectiveness. ${ }^{7}$

It is especially important to record true urine output by documenting amount of total output (urine plus irrigant) minus irrigant infused. ${ }^{7}$ Initiation of CBI does not guarantee against new clot formation, so nurses should still continue hourly monitoring and observe the need for intermittent manual irrigation, only extending this interval when the patient appears to be clot free. Reportable conditions pertinent at end of life include unresolved or increased pain or bladder spasms, bladder distention, dislodged or obstructed catheters. ${ }^{7}$ If clot formation appears to be minimal and intermittent, a trial of observation for spontaneous voiding may be permissible. If spontaneous voiding proves challenging or unreliable, a single-lumen standard Foley catheter, or a Coudé-tipped catheter when prostatic hypertrophy is anticipated, is reasonable.

In hospice and palliative medicine, where implementation of invasive measures is always balanced against a goal of maximizing comfort, even routine placement of an indwelling catheter deserves reflection as to how to minimize additional distress to the patient. In the emergency room setting, patients reported bladder catheterization to be one of the most painful routine procedures. ${ }^{8}$ In the post-anesthesia care unit setting, male gender and catheter diameter larger than ${ }^{18} \mathrm{Fr}$ were independent predictors of moderate or severe catheter-related bladder discomfort (CRBD). ${ }^{9}$ In the emergency room setting, females with preexisting pain experienced significantly higher discomfort during urethral catheterization. ${ }^{10}$ 
Many patients with advanced illnesses frequently have anatomic anomalies to their GU tract from their underlying disease processes or from treatment, particularly radiation or surgical interventions. Although supporting studies are scarce, intraurethral injection of lidocaine $2 \%$ gel prior to catheter insertion represents a low cost, low risk measure that may alleviate procedure-related discomfort. ${ }^{11}$ While relief from the deep visceral pain of urinary retention is always desirable, indwelling catheters may be symptomatically difficult to tolerate. Some experts recommend a trial of belladonna and opium suppositories, administered rectally three to four times daily as needed. Although studied only in small surgical populations thus far, visceral discomfort from indwelling catheters alone may be significantly relieved with oxybutynin or tolterodine, but likely not phenazopyridine. ${ }^{12,13}$

With greater or more frequent clot formation and retention, bladder irrigation becomes indicated, and insertion of a two- or three-way Foley catheter is prudent. Initially, aggressive hand irrigation with normal saline should be employed towards breaking up and removing clot. If urine color rapidly clears, this can be followed by observation in the hopes that the hematuria was self-limited. If conservative measures fail, then intermittent or continuous bladder irrigation (CBI) with normal saline should be initiated to prevent further clot formation. ${ }^{4}$

A number of adjunctive therapies have been employed in hopes of better control of hemorrhage and may be implemented as indicated by anatomy of bleeding source, location of care, and the patient's therapeutic goals. If the prostate is implicated, a trial of a $30 \mathrm{~mL}$ Foley balloon with gentle traction may facilitate tamponade; additionally, administration of alpha-5-reductase inhibitors may lessen bleeding. ${ }^{14}$ For bladder sources of bleeding, aminocaproic acid, an inhibitor of fibrinolytic enzymes, can be given orally or intravenously, or it can be administered intravesically during CBI, promoting resolution of hematuria in over $90 \%$ cases if given for 24 hours. ${ }^{15}$ However, side effects may include thromboembolic events when given systemically, or large intravesical clots when given during CBI. Other agents that can be infused during CBI include alum, silver nitrate, and formalin, each of which requires operator experience due to potential complications. Finally, intravesically infused hyperbaric oxygen successfully controls hemorrhagic cystitis in 70$80 \%$ cases, probably by promoting oxygenation of bladder mucosa and local vasoconstriction; however, this generally requires at least 30 treatments. ${ }^{16}$

Although only investigated in small studies, selective embolization of the internal iliac arteries (for bladder hemorrhage) or prostate bed (for prostate hemorrhage) may present a relatively low-risk option for control in palliative patients. ${ }^{17,18}$ When hematuria originates in the upper GU tract, arteriography and embolization may be necessary to stop hemorrhage. Management of ureteral outlet obstruction may require percutaneous nephrostomy tube placement to relieve hydronephrosis. In all cases, control of massive hemorrhage may necessitate endoscopic cauterization of lower GU lesions or surgical resection of prostate, bladder, or kidney.

The implementation of bladder irrigation can create great discomfort and distress, primarily from bladder detrusor muscle spasm. To date, there are no evidence-based guidelines and very few investigations regarding management of pain in this setting. In one study, 
administration of oral diazepam (4mg every 8 hours) during postoperative CBI significantly improved pain and awareness of bladder filling in patients undergoing transurethral resection of the prostate (TURP) for prostatic hypertrophy. ${ }^{19}$ In general, patients will almost certainly require opiate therapies for moderate-to-severe visceral pain, individualized in dose, frequency, and route of administration.

Case (Part III): The inpatient hospice physician and nurse met with Mr. J.R.'s wife to discuss options for managing the patient's gross hematuria and to reassess goals of care. The physician and nurse explained that further workup or invasive intervention should involve urologic consultation in a hospital setting; on the other hand, the inpatient hospice could continue bladder irrigation to attempt to relieve distress from ongoing bleeding, as well as continued expert symptom management. They explained that it seemed likely the bleeding would continue until enough clot formed and that maintaining urine flow would remain a challenge. They also explained that this persistent discomfort might escalate and therefore necessitate administration of escalating doses of opiates and/or benzodiazepines to alleviate distress.

At the bedside of patients with progressive gross hematuria, nurses and physicians alike are continually challenged to find the best balance between promoting comfort and staving off persistent hemorrhage. Foregoing a thorough workup and intervention may commit the patient to great distress from clot formation and bladder distention en route to acute kidney injury. On the other hand, aggressive management is itself invasive and painful, without certainty that the underlying etiology can be controlled. For the patient with a better functional status and focus on disease-modifying therapies, immediate transfer to an acute care setting for urological consultation is appropriate. Those with severely advanced disease or poor performance status may be less likely to tolerate the more invasive workup and management of gross hematuria outlined above.

Even in the hospice setting, where attention to pain relief is paramount, clinical care must be individualized. Patients should be thoroughly counseled as to risks/benefits of each intervention, and goals of care should be reevaluated often. Nursing staff may collaborate with their palliative physicians or hospice medical directors to determine whether urological consultation at home, the emergency room, or the inpatient hospice/palliative unit setting is available and indicated. For example, expert placement of a bladder catheter in the setting of abnormal lower GU anatomy, or rapid cystoscopy to visualize and cauterize intravesical lesions, may prove beneficial for optimizing symptom control without necessitating a hospital admission. As time permits, the entire hospice and palliative interdisciplinary team should take steps to assess the impact of this medical complication on the patient's qualityof-life, independence, and terminal trajectory.

Case (Part IV): After some consideration and discussion with family members, Mr. J.R.'s wife concluded that, under these circumstances, he would not have wanted to be transferred to another acute care facility for a more intensive workup or for invasive procedures. The inpatient hospice team changed from intermittent to continuous bladder irrigation to manage ongoing bleeding, with moderate improvement in urine outflow. Mr. J.R. continued to require escalating doses of 
parenteral opiates for pain management. His agitation increased but responded to benzodiazepines, eventually requiring a midazolam continuous infusion. The family and team agreed his care should remain at the inpatient hospice facility for best symptom management. He died comfortably 2 days later.

For the bedside HPM clinician, expert assessment and management of gross hematuria demands a confluence of skills in history-taking and physical assessment, symptom relief, communication, and collaboration with non-HPM care providers. When GU tract bleeding persists, medical interventions must always be balanced by the patient's overall goals of care.

\section{Acknowledgments}

The authors would like to thank Dr. Eric A. Singer for his input and expertise.

\section{References}

1. Gadler T, Keedy M, Rivas N. A case of hematuria. Adv Emerg Nurs J. 2010; 32(1):30-41.

2. Avidor Y, Nadu A, Matzkin H. Clinical significance of gross hematuria and its evaluation in patients receiving anticoagulant and aspirin treatment. Urology. 2000; 55(1):22-4. [PubMed: 10654888]

3. O'Connor OJ, McSweeney SE, Maher MM. Imaging in hematuria. Radiol Clin North Am. 2008; 46(1):113. [PubMed: 18328883]

4. Singer, EA., et al. Urologic issues in palliative care. In: Berger, A., et al., editors. Principles and Practice of Palliative Care and Supportive Oncology. 4. New Haven: Lippincott; In press

5. Aaronson DS, Walsh TJ, Smith JF, et al. Meta-analysis: does lidocaine gel before flexible cystoscopy provide pain relief? BJU Int. 2009 Aug; 104(4):506-9. [PubMed: 19239453]

6. Losco G, Antoniou S, Mark S. Male flexible cystoscopy: does waiting after insertion of topical anaesthetic lubricant improve patient comfort? BJU Int. 2011; 108(s2):42-44. [PubMed: 22085126]

7. Johnson, K.; Thomas, K.; Douglas, T. Continuous bladder irrigation for oncology patients: A proposed protocol. Virginia Henderson International Nursing Library; 2007. Retrieved 12/15/2011, from: http://hdl.handle.net/10755/165019

8. Singer AJ, Richman PB, Kowalska A, Thode HC Jr. Comparison of patient and practitioner assessments of pain from commonly performed emergency department procedures. Ann Emerg Med. 1999; 33:652-658. [PubMed: 10339680]

9. Binhas M, Motamed C, Hawajri N, Yiou R, Marty J. Predictors of catheter-related bladder discomfort in the post-anaesthesia care unit. Ann Fr d'Anesthesie et de Reanimation. 2011; 30:122125.

10. Tanabe P, Steinmann R, Anderson J, et al. Factors affecting pain scores during female urethral catheterization. Acad Emerg Med. 2004 Jun; 11(6):699-702. [PubMed: 15175213]

11. Siderias J, Guadio F, Singer AJ. Comparison of topical anesthetics and lubricants prior to urethral catheterization in males: a randomized controlled trial. Acad Emerg Med. 2004 Jun; 11(6):703-6. [PubMed: 15175214]

12. Agarwal A, Dhiraaj S, Singhal V, et al. Comparison of efficacy of oxybutynin and tolterodine for prevention of catheter related bladder discomfort. Br J Anaesth. 2006; 96(3):377-80. [PubMed: 16415311]

13. Anderson C, Chimhanda M, Sloan J, et al. Phenazopyridine does not improve catheter discomfort following gynecologic surgery. Am J Obstet Gynecol. 2011 Mar; 204(3):267.e1-3. [PubMed: 21376167]

14. Foley SJ, Soloman LZ, Wedderburn AW, et al. A prospective study of the natural history of hematuria associated with benign prostatic hyperplasia and the effect of finasteride. J Urol. 2000; 163(2):496-8. [PubMed: 10647664] 
15. Singh I, Laungani GB. Intravesical epsilon aminocaproic acid in management of intractable bladder hemorrhage. Urology. 1992; 40(3):227-9. [PubMed: 1523745]

16. Corman JM, McClure D, Pritchett R, et al. Treatment of radiation induced hemorrhagic cystitis with hyperbaric oxygen. J Urol. 2003; 169(6):2200-2. [PubMed: 12771749]

17. Liguori G, Amodeo A, Mucelli FP, et al. Intractable haematuria: long-term results after selective embolization of the internal iliac arteries. BJU Int. 2010; 106(4):500e503. [PubMed: 20128777]

18. Rastinehad AR, Caplin DM, Ost MC, et al. Selective arterial prostatic embolization (SAPE) for refractory hematuria of prostatic origin. Urology. 2008; 71(2):181e184. [PubMed: 18308078]

19. Nott MR, Jameson PM, Julious SA. Diazepam for relief of irrigation pain after transurethral resection of the prostate. Eur J Anaesth. 1997; 14:197-200. 


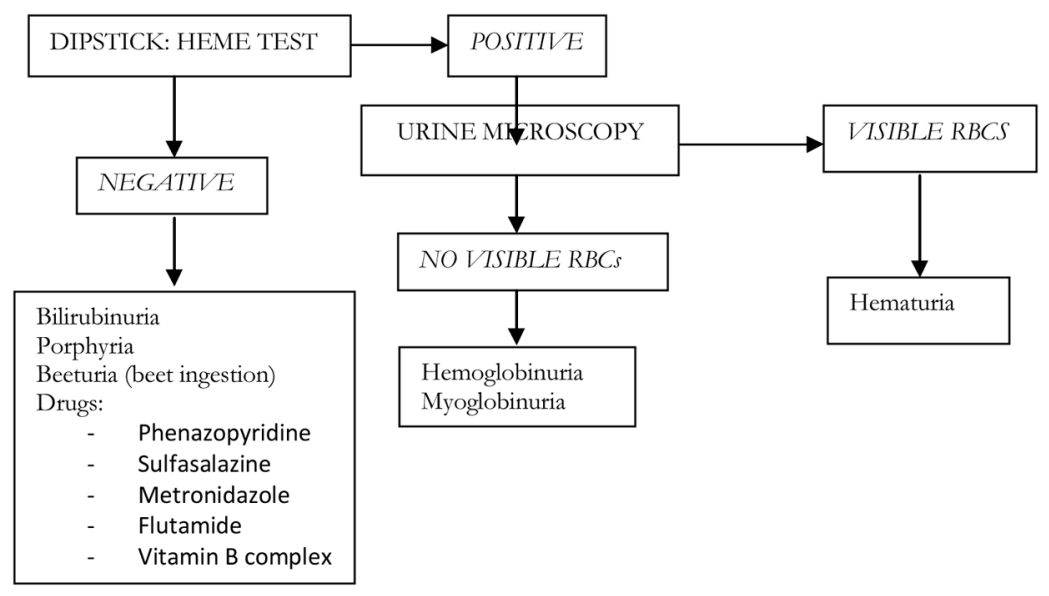

FIGURE 1. URINALYSIS (DIPSTICK AND MICROSCOPY) OF RED OR BROWN-TINGED URINE

Adapted from Feldman AS, Hsu C, Kurtz M, et al. Etiology and evaluation of hematuria in adults. In: UpToDate, Basow, DS (Ed), UpToDate, Waltham, MA, 2012. 
TABLE 1

\section{SOURCES OF HEMATURIA ALONG THE GENITOURINARY TRACT}

RENAL:

- Renal mass:

- Benign: angiomyolipoma, oncocytoma, abscess

- Malignant: renal cell carcinoma, transitional cell carcinoma

- Glomerular bleeding (e.g. IgA nephropathy, Alport syndrome)

- $\quad$ Structural disease (polycystic kidney disease, medullary sponge kidney)

- $\quad$ Pyelonephritis

- Hydronephrosis/distension

- Hypercalciuria, hyperuricosuria

- Malignant hypertension

- Renal vein thrombosis, renal artery occlusion

- Arteriovenous malformation

- $\quad$ Papillary necrosis (sickle cell disease)

- Trauma

\section{URETERAL:}

- Trauma

- Malignancy

- Nephrolithiasis

- $\quad$ Stricture

\section{BLADDER:}

- Malignancy (transitional cell carcinoma, squamous cell carcinoma)

- Radiation

- Cystitis (infection, cyclophosphamide, ifosphamide)

\section{PROSTATE/URETHRA:}

- Malignancy

- $\quad$ Benign prostatic hypertrophy

- Trauma (including traumatic catheterization)

- Urethritis

- Urethral diverticulum

Adapted with permission from Turner B. Hematuria: causes and management. Nurs Stand 2008. 23(1):50-56. 
TABLE 2

HISTORICAL AND PHYSICAL FINDINGS IN GROSS HEMATURIA
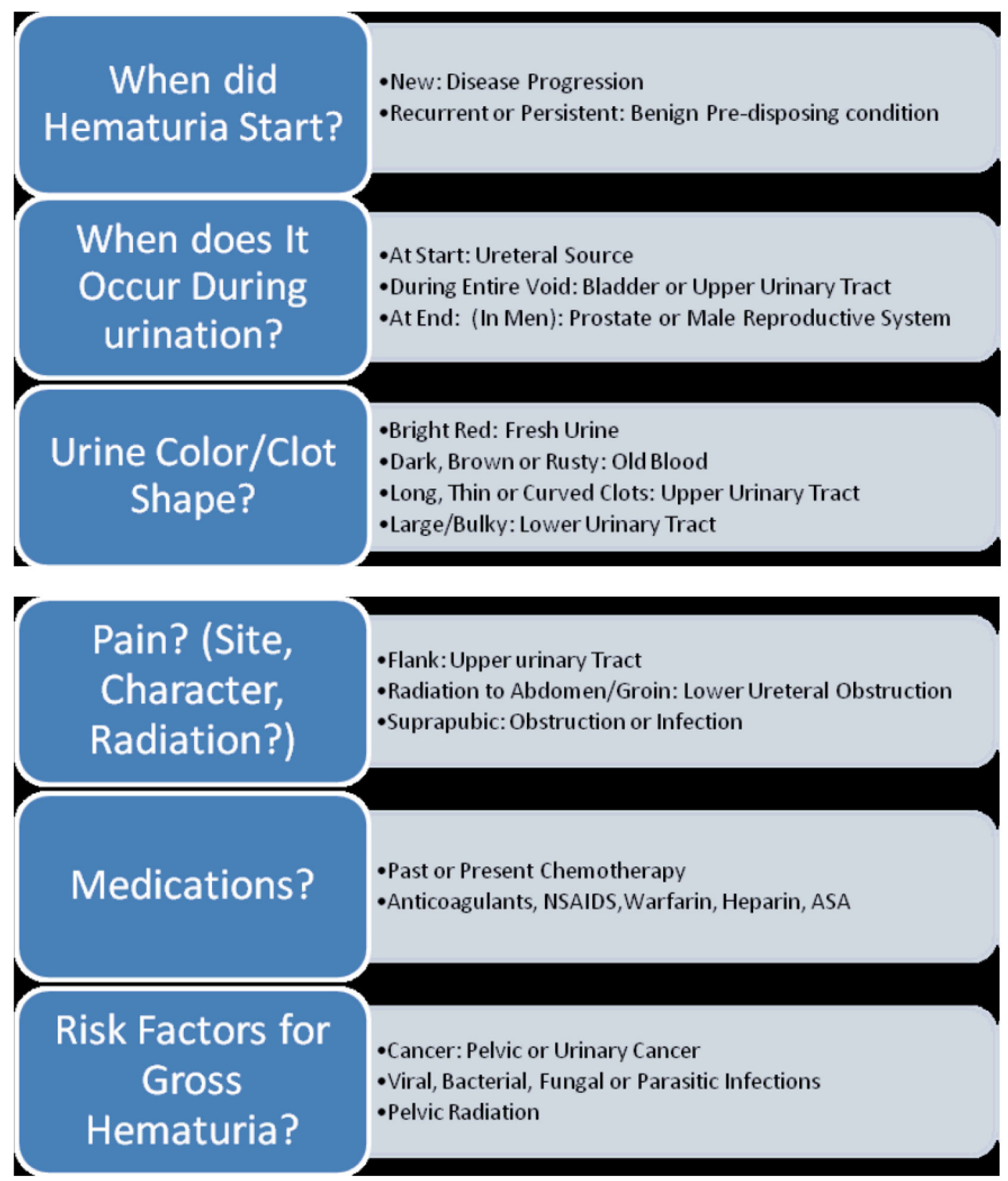


\section{Significant $\cdot$ Bladder Distention}

-Abdominal, Rectal or Prostate Masses Physical

- New Hypertension: New Onset or Rapid Increase=

Possible Renal Source Findings?

- Skin Rashes: Vasculitis

- Bruises, Purpura: Potential Coagulation Disorders

Adapted with permission from: Gray, M., \& Campbell, F. (2006). Urinary tract disorders. In BR Ferrell \& N Coyle (Eds.), Textbook of palliative nursing (2nd ed., pp. 265-283). New York, New York: Oxford University Press, Inc. 
TABLE 3

\section{NURSING IMPLICATIONS FOR ASSESSING AND MANAGING GROSS HEMATURIA}

- $\quad$ Obtain a detailed history and physical

- Understand etiology of hematuria to assess severity, impact and potential interventions

- Understand potential medical interventions to assess or intervene in gross hematuria at end of life

- Continuously assess severity of bleeding and urinary tract patency: obstruction is considered an end of life emergency

- Understand and perform nursing principles and practices of intermittent and continuous bladder irrigation

- Understand rationale behind various pharmacologic and topical measures to manage catheter related pain or discomfort, hemorrhage

- Consider the impact of changing the current care setting to obtain urologic consultation on patient comfort, quality of life, and goals of care

- $\quad$ Promote open communication and clarification of patient/family goals

- Coordinate with medical team and members of the interdisciplinary team to discuss and assess the impact of gross hematuria on patient quality of life, independence and terminal trajectory 